\title{
CORRECTION
}

\section{Correction to: Genome-wide analysis of the MYB-CC gene family of maize}

\author{
Jianrong Bai ${ }^{1}$ Fengjie Sun ${ }^{2} \cdot$ Minglei Wang ${ }^{3} \cdot$ Liang Su $^{1} \cdot$ Rui Li $^{1} \cdot$ Gustavo Caetano-Anollés ${ }^{3}$ (D)
}

Published online: 31 October 2018

(c) Springer Nature Switzerland AG 2018

\section{Correction to: Genetica \\ https://doi.org/10.1007/s10709-018-0042-y}

In the original publication of the article, the incorrect version of Fig. 1 was mistakenly used. The correct version of Fig. 1 is provided in this correction.

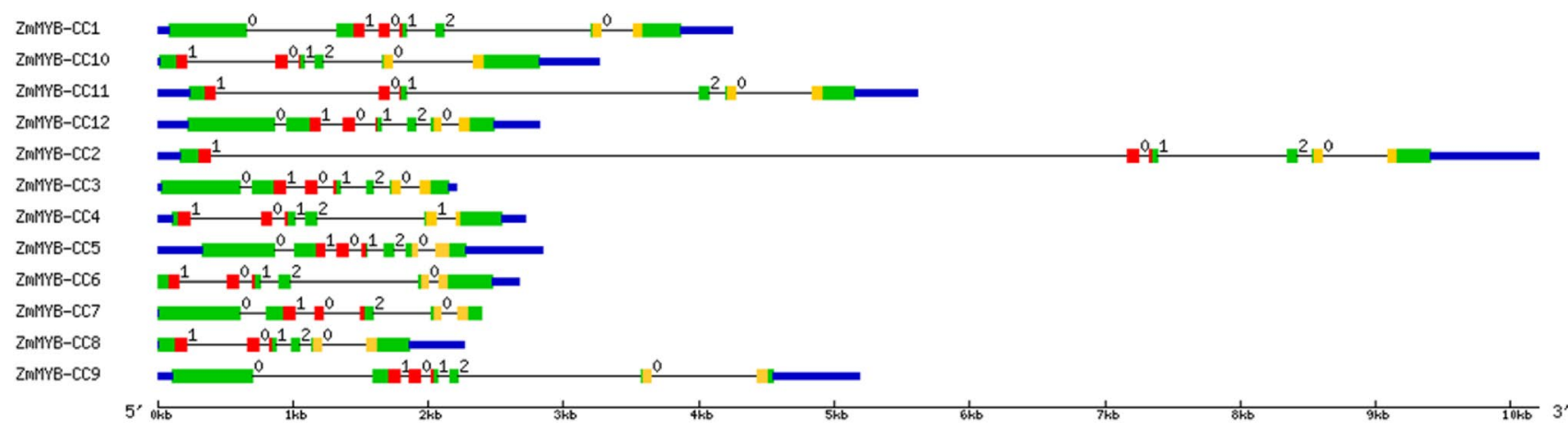

Legend:

exon MYB domain $\mathrm{CC}$ domain - intron upstream/downstream 012 : intron phase

Fig. 1 Gene structure of the maize $M Y B-C C$ family. Exons and introns are illustrated with filled boxes and single lines, respectively. Conserved sequences of MYB and CC proteins are marked in red and yellow, respectively. UTRs are displayed using thick blue lines at both ends of the sequence. Intron phases 0,1 , and 2 are indicated with 0 , 1 , and 2 , respectively

The original article can be found online at https://doi.org/10.1007/ s10709-018-0042-y.

Gustavo Caetano-Anollés

gca@illinois.edu

1 Institute of Crop Sciences, Shanxi Academy of Agricultural Sciences, Taiyuan 030031, Shanxi, China

2 School of Science and Technology, Georgia Gwinnett College, Lawrenceville, GA 30043, USA

3 Department of Crop Sciences, University of Illinois at Urbana-Champaign, Urbana, IL 61801, USA 